\title{
Diagnosis of Diabetes Mellitus
}

\author{
Ngugi M $P^{1, *}$, Njagi $J M^{2}$, Kibiti $C M^{3}$, Ngeranwa $J \mathbf{J} \mathbf{N}^{1}$, Njagi E $\mathbf{N} M^{1}$ \\ ${ }^{1}$ Department of Biochemistry and Biotechnology, Kenyatta University, Nairobi, P.O Box 43844-00100, Kenya \\ ${ }^{2}$ Department of Environmental Health, Kenyatta University, Nairobi, P.O Box 43844-00100, Kenya \\ ${ }^{3}$ Department of Pure and Applied Sciences, Mombasa Polytechnic University College, Mombasa, P.O Box 90420-80100, Kenya
}

\begin{abstract}
Diabetes mellitus is suspected based on symptoms. Urine and blood tests can be used to confirm a diagnosis of diabetes based on the amount of glucose in the urine and blood. Urine tests can also detect ketones and protein in the urine, which may help diagnose diabetes and assess how well the kidneys are functioning. These tests can also be used to monitor the disease once the patient is under treatment. This paper reviews the different diagnostic tests for diabetes mellitus.
\end{abstract}

Keywords Diabetes Mellitus, Ketones, Urine Tests, Blood Tests

\section{Introduction}

Diabetes Mellitus is a metabolic disturbance characterised by hyperglycaemia and a relative lack, or complete absence of insulin[1]. It is a disease, which by virtue of its complications may affect all organ systems in the body.

Prevention, timely diagnosis, and treatment are important in patients with diabetes mellitus. Many of the complications associated with diabetes, such as nephropathy, retinopathy, neuropathy, cardiovascular disease, stroke, and death, can be delayed or prevented with appropriate treatment of elevated blood pressure, lipids, and blood glucose[2,3].

The body usually is able to keep glucose concentrations stable. The normal fasting blood sugar is usually between $3.5-6.7 \mathrm{mmol} / 1$. After a meal it would rarely exceed $8 \mathrm{mmol} / 1$. Normally there is no glucose in urine since the normal threshold above which glucose would appear in the urine would be $10 \mathrm{mmol} / 1$. Below a concentration of $10 \mathrm{mmol} / 1$ the kidneys reabsorb glucose back into the blood stream and so glucose does not appear in the urine unless the blood concentration of glucose is high[4].

\section{Diagnostic Tests for Diabetes Mellitus}

\subsection{Urine Tests}

Urine tests are undertaken to analyse ketones bodies, glucose and proteins in the urine. The colorimetric reaction that occurs between ketones and nitroprusside (Sodium nitroferricyanide) is the method used for the rapid semiquantitive measurements of ketones $[5,6]$.

* Corresponding author:

matpiero@gmail.com (Ngugi MP)

Published online at http://journal.sapub.org/diabetes

Copyright (C) 2012 Scientific \& Academic Publishing. All Rights Reserved
Clinistix ${ }^{\circledR}$ and Diastix ${ }^{\circledR}$ are paper strips or dipsticks that change color when dipped in urine. The test strip is compared to a chart that shows the amount of glucose in the urine based on the change in color. The level of glucose in the urine lags behind the level of glucose in the blood. Testing the urine with a test stick, paper strip, or tablet is not as accurate as blood testing. However, it can give a fast and simple reading. Ketones in the urine can be detected using similar types of dipstick tests (Acetest or Ketostix)[7]. Urine samples with a specific gravity of 1.010 to 1.020 yield the most accurate results[8].

Ketoacidosis can be a life-threatening situation in Type I diabetics, so having a quick and simple test to detect ketones can assist in establishing a diagnosis sooner. Another dipstick test can determine the presence of protein or albumin in the urine. Protein in the urine can indicate problems with kidney function and can be used to track the development of renal failure. A more sensitive test for urine protein uses radioactively tagged chemicals to detect microalbuminuria, small amounts of protein in the urine, which may not show up on dipstick tests[7].

\subsection{Blood Tests}

Various blood test methods are routinely used in diagnosis of diabetes mellitus. They are discussed here below.

\subsubsection{Fasting Glucose Test}

Fasting glucose is directly proportional to the severity of the diabetes mellitus. During this test, blood is drawn from a vein in the patient's arm after the patient has not eaten for at least eight hours, usually in the morning before breakfast. The red blood cells are separated from the sample and the amount of glucose is measured in the remaining plasma. A plasma level of $200 \mathrm{mg} / \mathrm{dL}$ or greater strongly indicates diabetes provided that drugs such as glucocorticoids are not being administered. The fasting glucose test is usually repeated on another day to confirm the results[8]. 
Diabetics who are under control exhibit wide variation in their plasma glucose concentrations. Plasma glucose levels in controlled diabetics range during a typical 24- hour period from as low as $250 \mathrm{mg} / \mathrm{L}$ to as high as $3250 \mathrm{mg} / \mathrm{L}$. These variations are wider than those of nondiabetics[9].

\subsubsection{Postprandial Plasma Glucose Test}

Diabetes mellitus is more readily detected when carbohydrate metabolic capacity is tested. This can be done by stressing the system with a defined glucose load. Measurement of the rate that the glucose load is cleared from the blood, as compared to the rate of glucose clearance in healthy persons, detects impairment in glucose metabolism. A meal high in carbohydrates is used as the carbohydrate load, although a $75 \mathrm{~g}$ glucose drink is usually preferred over a meal. Blood is drawn at 2 hours after ingestion of the meal or glucose drink. Glucose levels above $1400 \mathrm{mg} / \mathrm{L}$ are abnormal; levels of 1200 to $1400 \mathrm{mg} / \mathrm{L}$ are ambiguous; and levels below $1200 \mathrm{mg} / \mathrm{L}$ are normal[7, 8].

Though widely used for detection of diabetes mellitus, this test method is highly inaccurate due to several variables that are difficult to control or adjust for. These variables include age, weight, previous diet, activity, illness, medications, time of the day that the test is conducted and actual size of the glucose dose. When a meal is used as the load, the effective glucose load depends on the digestion of disaccharides and polysaccharides and their subsequent absorption from the intestinal tract[8].

\subsubsection{Oral Glucose Tolerance Test (OGTT)}

The oral glucose tolerance test evaluates clearance from the circulation after glucose loading under defined and controlled conditions. The test has been standardized by the Committee on Statistics of the American Diabetes Association[10]. The patient should have been fasting for the previous $8-14$ hours. A zero time (baseline) blood sample is drawn. The patient is given a glucose solution, which is drunk within 5 minutes. Blood is drawn at intervals for measurement of glucose (blood sugar), and sometimes insulin levels[7].

The intervals and number of samples vary according to the purpose of the test. For simple diabetes screening, the most important sample is the 2-hour sample. The zero and 2-hour samples may be the only ones collected. In a non-diabetic, the level of glucose in the blood goes up immediately after the drink and then decreases gradually as insulin is used by the body to metabolize or absorb the sugar. In a diabetic, the glucose in the blood goes up and stays high after drinking the sweetened liquid. A plasma glucose level of $2000 \mathrm{mg} / \mathrm{L}$ or higher at two hours after drinking the syrup and at one other point during the two-hour test period confirms the diagnosis of diabetes[7].

During the test, the patient must be ambulatory, since inactivity decreases glucose tolerance. The test can also be affected by illness, abnormalities of such hormones as thyroxine, growth hormone, cortisol, and catecholamines, drugs and medications such as oral contraceptives, salicylates, nicotinic acid, diuretics and hypoglycaemic agents and testing time. The glucose load should consist of glucose only[11].

\subsubsection{Intravenous Glucose Tolerance Test}

The intravenous glucose tolerance test is used for persons with malabsorptive disorders or previous gastric or intestinal surgery. Glucose is administered intravenously over 30 minutes, using a $20 \%$ solution. A glucose load of $0.5 \mathrm{~g} / \mathrm{kg}$ of body weight is used. Non-diabetics respond with a plasma glucose level of 2000 to $2500 \mathrm{mg} / \mathrm{L}$. Discontinuation of the glucose loading leads to a decrease in plasma levels with fasting levels reached at about 90 minutes. Diabetics demonstrate plasma glucose level above $2500 \mathrm{mg} / \mathrm{L}$ during administration of the load. On discontinuation of the loading, plasma glucose levels of diabetics also return to fasting levels at about 90 minutes. An alternative procedure called the Soskin method uses $50 \%$ glucose delivered intravenously within 3 to 5 minutes. The glucose load used is $0.3 \mathrm{~g} / \mathrm{kg}$ of body weight. Non- diabetics re-establish fasting levels in less than 60 minutes after discontinuing the glucose infusion. In diabetics fasting levels are established significantly later than 60 minutes[8].

\subsubsection{O'Sullivan Test}

This test is used to detect gestational diabetes. A $50 \mathrm{~g}$ load of glucose is given to a fasting patient. Blood is drawn at one hour. Gestational diabetes is suggested by plasma levels above $1500 \mathrm{mg} / \mathrm{L}[8,12]$.

\subsubsection{Glycated Hemoglobin and Plasma Albumin Test}

In diabetes mellitus, a minor hemoglobin derivative called $\mathrm{HbAlc}$ is produced by glycosylation. Sine this reaction is spontaneous and since erythrocytes are completely permeable to glucose, the quantity of $\mathrm{HbAlc}$ formed is directly proportional to the average plasma glucose concentration that the erythrocytes are exposed to during their 120-day life span (4 to 6 weeks before sampling). For normoglycemic persons, HbA1c constitutes $4 \%$ to $5 \%$ of total hemoglobin, whereas in diabetics, $\mathrm{HbAl}$ c levels are significantly elevated. The elevations are directly proportional to the long-term degree of hyperglycemia[13]. Glycated hemoglobins are the most useful for monitoring of diabetes mellitus. However, they are not sufficiently sensitive to effectively detect borderline cases of diabetes mellitus[14]. Serum albumin is also glycosylated to a degree proportional to plasma glucose levels. The short half-life for albumin of 15 days makes it a good monitor of short-term blood plasma glucose levels[15].

\subsubsection{Plasma insulin Test}

Fasting plasma insulin levels in type I diabetics are low. Those of type II diabetics are low only when fasting plasma glucose levels exceed $2500 \mathrm{mg} / \mathrm{L}$. Otherwise, they are normal. A glucose challenge separates type I diabetics from type II diabetics. Glucose loading elicits no significant insulin re- 
sponse for type I diabetics and a delayed, exaggerated response in type II diabetics[16].

Diabetics can monitor their own blood glucose levels with home blood glucose monitoring kits. A small needle or lancet is used to prick the finger and a drop of blood is collected and analyzed by a monitoring device. The correct use of such a device minimizes the wide variations of blood glucose experienced by diabetics and, as a result, the hypoglycemic events and even the long-term complications of diabetes mellitus. Some patients may test their blood glucose levels several times during a day and use this information to adjust their diet or doses of insulin[17].

In a consensus statement on blood glucose monitoring, many insulin-treated populations have been recommended for self-monitoring programmes. These include pregnant women, patients with unstable diabetes, patients with histories of severe ketosis or hypoglycemia especially those who do not demonstrate warning symptoms of hypoglycemia, patients receiving intensive insulin therapy and patients with abnormal renal thresholds for glucose[18].

\section{Conclusions}

The diagnostic criterion for the diagnosis of diabetes mellitus has been undergoing review globally. The American Diabetes Association (ADA) and the World Health Organization (WHO) have always released differing recommendations regarding the criteria for diagnosis of diabetes mellitus. For instance, in 2003 the ADA modified its recommendations resulting in discrepancies between its recommendations and those of the WHO.

The disparities emanate from the difference in fasting plasma glucose levels for defining Impaired Fasting Glucose (IFG), inclusion of $2-\mathrm{h}$ plasma glucose value in defining IFG, and inclusion of $2-\mathrm{h}$ plasma glucose value in defining IFG. These disparities may result in differences in an individual's classification of glucose tolerance. As ADA recommends fasting plasma glucose as the recommended method for diagnosing asymptomatic diabetes, WHO recommends the oral glucose tolerance test.

These discrepancies have implications for the individual and for population prevalence estimates. For example people who fall into the ADA category of IFG could include people with IGT or diabetes if a 2-h plasma glucose is not measured, and ADA defined IGT could include diabetes if a fasting plasma glucose is not measured.

It is also worth mentioning that the criteria for diagnosis of diabetes mellitus should be formulated taking into account the populations living in different parts of the world. This idea is informed by the fact that different populations residing in different parts of the world have varying thresholds of physiological parameters. In this regard, the existing criteria may only be useful strictly for the populations tested during the formulation of the criteria. They may only serve to provide general guidelines regarding diagnosis of diabetes mellitus.
In conclusion, various methods based on urine and blood tests are available for diagnosis of diabetes mellitus. The values for that confirm diagnosis of diabetes mellitus may differ from from one individual to another. However, there are general threshold values that importantly provide guidelines on the diagnosis of diabetes mellitus. These guidelines are regularly reviewed by the $\mathrm{WHO}$ and $\mathrm{ADA}$, owing to emerging trends in diagnosis.

\section{REFERENCES}

[1] R. Souhami, J. Moxham, Diabetes Mellitus and disorders of lipid and intermediary metabolism. Churchill Livingston, London, 1994.

[2] American Diabetes Association, "Standards of medical care in diabetes- 2010”, Diabetes Care, vol. 33(1), pp. S11-S61, 2010 .

[3] UK Prospective Diabetes Study (UKPDS) Group, "Intensive blood-glucose control with sulphonylureas or insulin compared with conventional treatment and risk of complications in patients with type 2 diabetes", Lancet, vol. 352(9131), pp. $837-853,1998$.

[4] DCCT Research Group, "The effect of intensive diabetes treatment on the development and progression of long term complication in insulin dependent diabetes mellitus: The Diabetes Control and Complications Trial", New England Journal of Medicine, vol. 329, pp. 978-986, 1993.

[5] T.E. Friedemann, B.B. Sheft, V.C. Miller, "An assessment of the value of nitroprusside reaction for the determination of ketone bodies in urine" Queens Bullettin of Northwestern University Medical School, vol. 20, pp. 301-310, 1946.

[6] P.L. Li, J.T. Lee, M.H. McGilliray, "Direct fixed-time kinetic assays for beta-hydroxybutyrate and acetoacetate with a centrifugal analyser or a computerbacked spectrophotometer" Clinical Chemistry, vol 26, pp 1713-1717, 1980.

[7] R. Belinda, Gale Encyclopaedia of Alternative Medicine 2004:2603-2605.

[8] A. K. Lawrence, J.P. Amadeo, Clinical Chemistry: Theory, analysis and correlation, 3rd Ed., St. Louis: Mosby Inc, USA, 1996.

[9] A.C. Mauer, "The therapy of diabetes", American Scientist, vol. 67, pp. 422-428, 1979.

[10] American Diabetes Association, "Standardization of the Oral Glucose Tolerance Test”, Diabetes, vol. 18, pp. 299-303, 1969.

[11] M.B. Davidson, "The effects of aging on carbohydrate metabolism: A review of the English literature and a practical approach to the diagnosis of diabetes mellitus in the elderly", Metabolism, vol. 28, pp. 688-693, 1979.

[12] M.P. Ngugi, "Hypoglycemic effects of some Kenyan plants used in management of diabetes mellitus in eastern province", MSc. thesis, Kenyatta University, Kenya, 2006.

[13] S. Larsen, J. Hilsted, E.K. Philipsen, B. Tronier, N.J. Christensen, Damkjaer M. Nielsen, H. Worning, "Glucose coun- 
terregulation in diabetes secondary to chronic Pancreatitis", Metabolism, vol. 39, pp. 138-143, 1990.

[14] R.F. Dods, C. Bolmey, "Glycosylated haemoglobin assay and oral glucose tolerance test compared for detection of diabetes mellitus", Clinical Chemistry, vol. 25, pp. 764-768, 1979.

[15] C.E. Guthrow, M.A. Morris, J.F. Day, "Enhanced nonenzymatic glycosylation of serum albumin in diabetes mellitus", Proceedings of National Academy of Science, USA, vol. 76, pp. 4528-4531, 1979.

[16] W.K. Ward, J.C. Beard, J.B. Halter, "Pathophysiology of insulin secretion in non- insulin dependent diabetes mellitus", Diabetes Care, vol. 7, pp. 491-497, 1984.

[17] R. Turner, C. Cull, R. Holman, "United Kingdom Prospective Diabetes Study 17: a 9-year update of a randomised, controlled trial on the effect of improved metabolic control on complications in non-insulin-dependent diabetes mellitus", Annals of Internal Medicine, vol. 124, no. 1, pp. 136-145, 1996.

[18] Consensus Development Panel, "Consensus Statement on Self-Monitoring of Blood Glucose", Diabetes Care, vol. 10, pp. 95-99, 1987. 